\title{
Intrinsic coherence resonance in excitable membrane patches
}

\author{
Gerhard Schmid *, Peter Hänggi \\ Institut für Physik, Universität Augsburg, Theoretische Physik I, D-86135 Augsburg, Germany
}

Received 22 May 2006; received in revised form 24 August 2006; accepted 28 August 2006

Available online 7 September 2006

\begin{abstract}
The influence of intrinsic channel noise on the spiking activity of excitable membrane patches is studied by use of a stochastic generalization of the Hodgkin-Huxley model. Internal noise stemming from the stochastic dynamics of individual ion channels does affect the electric properties of the cell-membrane patches. There exists an optimal size of the membrane patch for which the internal noise alone can cause a nearly regular spontaneous generation of action potentials. We consider the influence of intrinsic channel noise in presence of a constant and an oscillatory current driving for both, the mean interspike interval and the phenomenon of coherence resonance for neuronal spiking. Given small membrane patches, implying that channel noise dominates the excitable dynamics, we find the phenomenon of intrinsic coherence resonance. In this case, the relatively regular spiking behavior becomes essentially independent of an applied stimulus. We observed, however, the occurrence of a skipping of supra-threshold input events due to channel noise for intermediate patch sizes. This effect consequently reduces the overall coherence of the spiking.

(C) 2006 Elsevier Inc. All rights reserved.
\end{abstract}

PACS: 87.10.+e; 87.16.-b; 05.45.Xt; 05.40.Ca

Keywords: Ion channels; Excitable dynamics; Channel noise; Coherence resonance

\footnotetext{
* Corresponding author. Tel.: +49 821598 3229; fax: +49 8215983222.

E-mail address: Gerhard.Schmid@physik.uni-augsburg.de (G. Schmid).
} 


\section{Introduction}

A prominent question in neurophysiology concerns the limiting factors of the reliability of neuronal responses to given stimuli. In this work we focus on a particular aspect of this intricate issue, namely the impact of channel noise, which is generated by random gating dynamics of the ion channels in membrane patches of finite size. Since the work of Lecar and Nossal $[1,2]$ it became clear, however, that not only the synaptic noise but also the randomness of the ion channel gating itself may cause threshold fluctuations in neurons [3]. Therefore, channel noise which stems from the stochastic nature of the ion channel dynamics must be taken into account [3]. It impacts such features as the threshold to spiking and the spiking rate itself [4-6], the anomalous noise-assisted enhancement of transduction of external signals, i.e., the phenomenon of Stochastic Resonance [7-12] and related, the efficiency for synchronization [13-15], to name but a few such interesting phenomena. The origin of the channel noise [3] is basically due to fluctuations of the mean number of open ion channels around the corresponding mean values. Therefore, the strength of the channel noise is mainly determined by the number of ion channels participating in the generation of action potentials.

In this paper we investigate within a stochastic generalization of the Hodgkin-Huxley model the influence of the channel noise on the mean interspike intervals and on the coherence of the spiking activity produced within the cell membrane containing a certain amount of ion channels. This is distinct from the effects of fluctuations due to synaptic noise which were studied within generalized Hodgkin-Huxley models [16,17]. The effect of coherence resonance describes a phenomena of self-synchronization of a system due to noise. It was observed for single excitable dynamics, e.g., for the Fitz Hugh-Nagumo model [18] or for a Hodgkin-Huxley model taking into account the influence of synaptic noise [16], and, as well as for neuronal networks [19,20].

\section{The Hodgkin-Huxley model}

Starting with the well-established model of Hodgkin and Huxley [21] we consider a cell-membrane patch of area $\mathscr{A}$ as an electrical capacitor possessing the specific area capacitance $C$. The membrane separates two ionic bath solutions (which in vivo correspond to the interior and the exterior of the excitable cell) with different concentrations of the ions of different sorts, mainly potassium, $\mathrm{K}^{+}$, sodium, $\mathrm{Na}^{+}$, and chloride, $\mathrm{Cl}^{-}$ions. The macroscopic concentration differences are kept constant. In the cell this task is accomplished by ATP-driven ionic pumps. Furthermore, the ionic baths are on the average electrically neutral. However, due to the different ionic concentrations on the opposite sides of the semi-permeable membrane, the membrane becomes charged. As a consequence, an equilibrium transmembrane electrical potential difference emerges. The lipid membrane creates an almost impenetrable barrier for the ions. However, they can flow across the membrane through special ion selective pores created by specialized membrane proteins - the ion channels [22]. The specific potassium, $I_{\mathrm{K}}$, and sodium, $I_{\mathrm{Na}}$, ion currents through the open ion channels are approximately proportional to the differences of the transmembrane potential $V$ and the specific (for the particular sort of ions) equilibrium potentials, $E_{\mathrm{K}}$ and $E_{\mathrm{Na}}$, respectively. The stochastically averaged, mean conductances, $G_{\mathrm{Na}}(m, h)$ and $G_{\mathrm{K}}(n)$, are, however, strongly nonlinear functions of $V$. This nonlinearity emerges due to the gating dynamics (see below). There 
exists also the leakage current $I_{\mathrm{L}}$. If the membrane is driven by the external current $I_{\text {ext }}(t)$, the sum of the specific ion currents and the capacitive current, $I_{\mathrm{C}}$, must be equal to $I_{\mathrm{ext}}(t)$ as a consequence of the charge conservation. Therefore, the equation for the transmembrane potential $V(t)$ reads

$$
C \frac{\mathrm{d}}{\mathrm{d} t} V+G_{\mathrm{K}}(n)\left(V-E_{\mathrm{K}}\right)+G_{\mathrm{Na}}(m, h)\left(V-E_{\mathrm{Na}}\right)+G_{\mathrm{L}}\left(V-E_{\mathrm{L}}\right)=I_{\mathrm{ext}}(t) .
$$

For a squid giant axon, the parameters in Eq. (1) read $E_{\mathrm{Na}}=50 \mathrm{mV}, E_{\mathrm{K}}=-77 \mathrm{mV}$, $E_{\mathrm{L}}=-54.4 \mathrm{mV}$, and $C=1 \mu \mathrm{F} / \mathrm{cm}^{2}$. Furthermore, the leakage conductance is assumed to be constant, $G_{\mathrm{L}}=0.3 \mathrm{mS} / \mathrm{cm}^{2}$. On the contrary, the sodium and potassium conductances are controlled by the voltage-dependent gating dynamics of single ion channels and are proportional to their respective numbers. These latter assumptions have been fully confirmed in the single-channel recordings by Neher, Sakmann, and colleagues which indeed have proven that ion channels undergo the opening-closing stochastic gating dynamics [23]. In the Hodgkin-Huxley model, the opening of the potassium ion channel is governed by four identical activation gates characterized by the opening probability $n$. The channel is open when all four gates are open. In the case of sodium channel, the dynamics is governed by the three independent, identical fast activation gates $(m)$ and an additional slow, so-termed inactivation gate $(h)$. The independence of the gates implies that the probability $P_{\mathrm{K}, \mathrm{Na}}$ of the occurrence of the conducting conformation is $P_{\mathrm{K}}=n^{4}$ for a potassium channel and $P_{\mathrm{Na}}=m^{3} h$ for a sodium channel, respectively. In the mean-field description, the macroscopic potassium and sodium conductances thus read

$$
G_{\mathrm{K}}(n)=g_{\mathrm{K}}^{\max } n^{4}, \quad G_{\mathrm{Na}}(m, h)=g_{\mathrm{Na}}^{\max } m^{3} h,
$$

where $g_{\mathrm{K}}^{\max }=36 \mathrm{mS} / \mathrm{cm}^{2}$ and $g_{\mathrm{Na}}^{\max }=120 \mathrm{mS} / \mathrm{cm}^{2}$ denote the maximal conductances (when all channels are open). The two-state, opening-closing dynamics of the gates is given by the voltage dependent opening and closing rates $\alpha_{x}(V)$ and $\beta_{x}(V)(x=m, h, n)$, i.e.,

$$
\begin{aligned}
& \alpha_{m}(V)=\frac{0.1(V+40)}{1-\exp [-(V+40) / 10]}, \\
& \beta_{m}(V)=4 \exp [-(V+65) / 18], \\
& \alpha_{h}(V)=0.07 \exp [-(V+65) / 20], \\
& \beta_{h}(V)=\{1+\exp [-(V+35) / 10]\}^{-1}, \\
& \alpha_{n}(V)=\frac{0.01(V+55)}{1-\exp [-(V+55) / 10]}, \\
& \beta_{n}(V)=0.125 \exp [-(V+65) / 80] .
\end{aligned}
$$

Hence, the dynamics of the opening probabilities for the gates are given by

$$
\dot{x}=\alpha_{x}(V)(1-x)-\beta_{x}(V) x, \quad x=m, h, n .
$$

The voltage equations (1) and (2) and the rate equations of the gating dynamics (3) and (4) define the original, purely deterministic Hodgkin-Huxley model [21] for the squid giant axon.

The dynamics of the Hodgkin-Huxley model exhibits a complex and rich behavior which sensitively depends on the model parameters. For the squid giant axon parameters, the corresponding, non-driven dynamics possesses a single fixed point and does not exhibit a spiking activity in 
the absence of external stimulus, $I_{\mathrm{ext}}(t)=0$. However, if a constant stimulus, $I_{\mathrm{ext}}$, is applied, the fixed point loses its stability with increasing strength $I_{\mathrm{ext}}$ upon $I_{\mathrm{ext}} \geqslant I_{2} \approx 9.763 \mu \mathrm{A} / \mathrm{cm}^{2}[24,25]$. For such a super-threshold current strength, the membrane exhibits a periodic spiking activity which reflects the presence of a stable limit cycle, see Fig. 1 . The period of the solution $\mathscr{T}$ is depicted in Fig. 2. It is either zero for the stable fixed point solution or assumes a certain value for the stable oscillatory solution at given driving strength. At the point $I_{2}$, where a sub-critical Hopf-bifurcation takes place, a jump of the period $\mathscr{T}$ occurs. Upon decreasing the driving current strength, the spiking dynamics still persists below the threshold for excitation, i.e., also for $I<I_{2}$, until the diminishing current reaches the sub-critical value $I_{1} \approx 6.26 \mu \mathrm{A} / \mathrm{cm}^{2}$. Below this value, the limit cycle loses stability and the spiking activity vanishes. In conclusion, for $I_{1}<$ $I_{\text {ext }}<I_{2}$, both the stable fixed point and the stable limit cycle can coexist.

For a sinusoidal driving, i.e., $I_{\text {ext }}(t)=A \sin (\Omega t)$, where $A$ denotes the amplitude and $\Omega$ the angular driving frequency, the dynamics becomes more intricate as compared to the case with a constant driving force $I_{\mathrm{ext}}$. What is most important for the generation of action potentials is

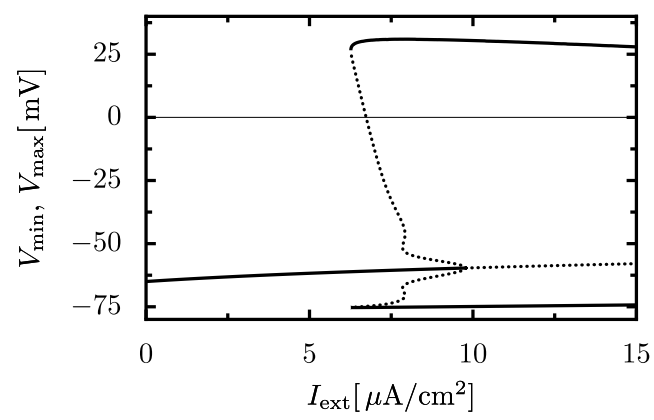

Fig. 1. The bifurcation diagram for the emergence of spiking behavior for the deterministic Hodgkin-Huxley model. Solid lines correspond to stable and dotted lines to unstable solutions. The equilibrium voltage (fixed point) and the minimal and maximal voltage amplitudes, respectively, of the limit cycle oscillations are plotted against the constant driving current strength. There exists a hysteretic behavior for the range $6.26<I_{\text {ext }}<9.763 \mu \mathrm{A} / \mathrm{cm}^{2}$ where the stable limit cycle exhibiting firing events and the stable fixed point coexist.

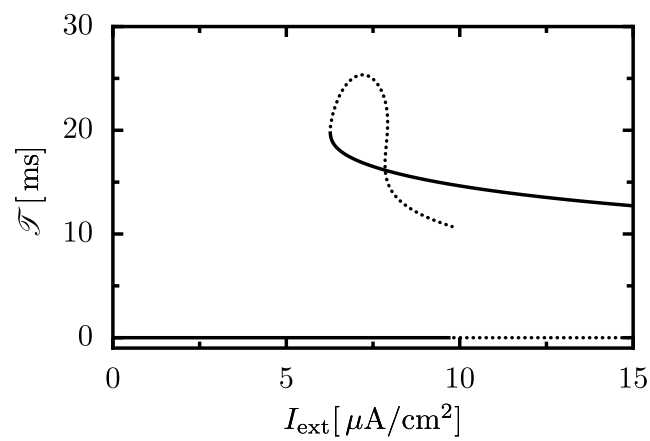

Fig. 2. Periodicity $\mathscr{T}$ of the solution of the driven Hodgkin-Huxley model (solid lines: stable solutions, dotted lines: unstable solutions). For force values larger than $6.26 \mu \mathrm{A} / \mathrm{cm}^{2}$ a stable periodic spiking solution exists, see in Fig. 1. A horizontal line at zero indicates the existence of a non-spiking solution. 
the frequency dependence of the threshold amplitude [26]. First systematic studies on this topic were performed by Aihara and collaborators [27].

\section{Stochastic generalization of Hodgkin-Huxley model}

Operating with an average number of open channels, disregards the corresponding number fluctuations, i.e., the so-called channel noise [3,23]. Therefore, the Hodgkin-Huxley model is valid, strictly speaking, only within the limit of a very large system size where these fluctuations can be neglected. In contrast, the role of internal fluctuations in membrane patches of finite size cannot be neglected a priori. In fact, there are studies which evidence unambiguously that the channel noise can be functionally important for the excitable dynamics $[3-5,9,10]$.

To account for the effect of channel noise, we use a generalization of the model introduced originally by Fox and $\mathrm{Lu}$ [28] which presents a stochastic generalization of the Hodgkin-Huxley equations (1-4). Due to the Kramers-Moyal expansion of the corresponding master equations, the dynamics of the gating variables is given by the following Langevin equation, interpreted here in the stochastic interpretation of Itô [29-31], reading

$$
\dot{x}=\alpha_{x}(V)(1-x)-\beta_{x}(V) x+\xi_{x}(t), \quad x=m, h, n
$$

driven by independent Gaussian white noise sources $\xi_{x}(t)$ of vanishing mean which take into account the fluctuations of the number of open gates. The (multiplicative) noise strengths depend on both, the membrane voltage and the gating variables. In precise terms, the noise correlations assume the following form for an excitable membrane patch with $N_{\mathrm{Na}}$ sodium and $N_{\mathrm{K}}$ potassium ion channels:

$$
\begin{aligned}
\left\langle\xi_{m}(t) \xi_{m}\left(t^{\prime}\right)\right\rangle & =\frac{2}{N_{\mathrm{Na}}} \frac{\alpha_{m}(V)(1-m)+\beta_{m}(V) m}{2} \delta\left(t-t^{\prime}\right), \\
\left\langle\xi_{h}(t) \xi_{h}\left(t^{\prime}\right)\right\rangle & =\frac{2}{N_{\mathrm{Na}}} \frac{\alpha_{h}(V)(1-h)+\beta_{h}(V) h}{2} \delta\left(t-t^{\prime}\right), \\
\left\langle\xi_{n}(t) \xi_{n}\left(t^{\prime}\right)\right\rangle & =\frac{2}{N_{\mathrm{K}}} \frac{\alpha_{n}(V)(1-n)+\beta_{n}(V) n}{2} \delta\left(t-t^{\prime}\right) .
\end{aligned}
$$

To ensure that at all times the confinement of the gating variables take on values between 0 (all gates are closed) and 1 (all gates are open) only, we implemented numerically reflecting boundaries at 0 and 1 . With the assumption of homogeneous ion channel densities, $\rho_{\mathrm{Na}}=$ $6 \times 10^{13} \mathrm{~m}^{-2}=60 \mu \mathrm{m}^{-2}$ and $\rho_{\mathrm{K}}=1.8 \times 10^{13} \mathrm{~m}^{-2}=18 \mu \mathrm{m}^{-2}$, the ion channel numbers are given by

$$
N_{\mathrm{Na}}=\rho_{\mathrm{Na}} \mathscr{A}, \quad N_{\mathrm{K}}=\rho_{\mathrm{K}} \mathscr{A} \text {. }
$$

\section{Noise-induced spontaneous spiking}

We investigate numerically the influence of channel noise for different external stimulation with respect of the coherence of the resulting spiking behavior. The numerical integration of Eqs. (1) and (5) is carried out by the standard Euler algorithm with the step size $\Delta t \approx 2 \times 10^{-3} \mathrm{~ms}$. The 
"Numerical Recipes" routine ran2 is used for the generation of independent random numbers [32] with the Box-Muller algorithm providing the Gaussian distributed random numbers. From the stochastic voltage signal $V(t)$ we extract a point process of spike occurrences $\left\{t_{i}\right\}$

$$
u(t):=\sum_{i=1}^{N} \delta\left(t-t_{i}\right)
$$

where $N$ is the total number of spikes occurring during the elapsed time interval. The occurrence of a spike in the voltage signal $V(t)$ is detected by upward-crossing a certain detection threshold value $V_{0}=0$. Notable, the threshold can be varied over a wide range with no effect on the resulting spike train dynamics.

For small internal noise strengths, i.e., large patch sizes, the occurrence of an action potential is very rare in the autonomous, non-driven regime: $I_{\mathrm{ext}}=0$, see Fig. 3 (bottom panel). Upon decreasing the patch size $\mathscr{A}$ we observe an increasing spiking activity due to the strengthening of internal channel noise, see Fig. 3.

The interspike intervals $T_{i}$ are given by the spike occurrences $\left\{t_{i}\right\}: T_{i}:=t_{i+1}-t_{i}$. A quantity which describes the spiking behavior more quantitatively is the mean interspike interval $\langle T\rangle$ which is the inverse spiking rate; i.e.,

$$
\langle T\rangle=\lim _{N \rightarrow \infty} \frac{1}{N} \sum_{i} T_{i} .
$$

Channel noise acting on the electric behavior of the cell-membrane patch determines the generation of action potentials. Some qualitative considerations show that with increasing channel noise the generation of action potentials raises for sub-threshold driving $\left(I_{\mathrm{ext}}<I_{1}\right)$. However, for a supra-threshold driving $\left(I_{\mathrm{ext}}>I_{2}\right)$, the skipping events gain influence and channel noise could suppress the generation of action potentials. Then, upon increasing the channel noise strength the spiking rate is reduced and the mean interspike interval starts rising, starting from the value for the deterministic case, see Fig. 4. Because for small patch sizes channel noise dominates the spike generation the mean interspike intervals become shorter as compared to the case of deterministic

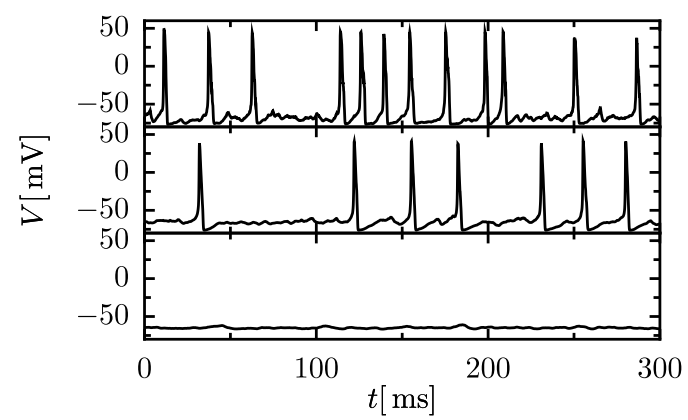

Fig. 3. Three simulated realization of the voltage dynamics in absence of external driving $\left(I_{\mathrm{ext}}=0\right)$ and for three patch sizes: top panel $\mathscr{A}=1 \mu \mathrm{m}^{2}$, middle panel $\mathscr{A}=16 \mu \mathrm{m}^{2}$, and bottom panel $\mathscr{A}=128 \mu \mathrm{m}^{2}$. With decreasing patch size (bottom-to-top) the fluctuations of the number of open ion channels gain influence; therefore more action potentials are produced for the smallest patch size, whereas for very large patch sizes the intrinsic channel noise strength is hardly capable of producing voltage spikes. 

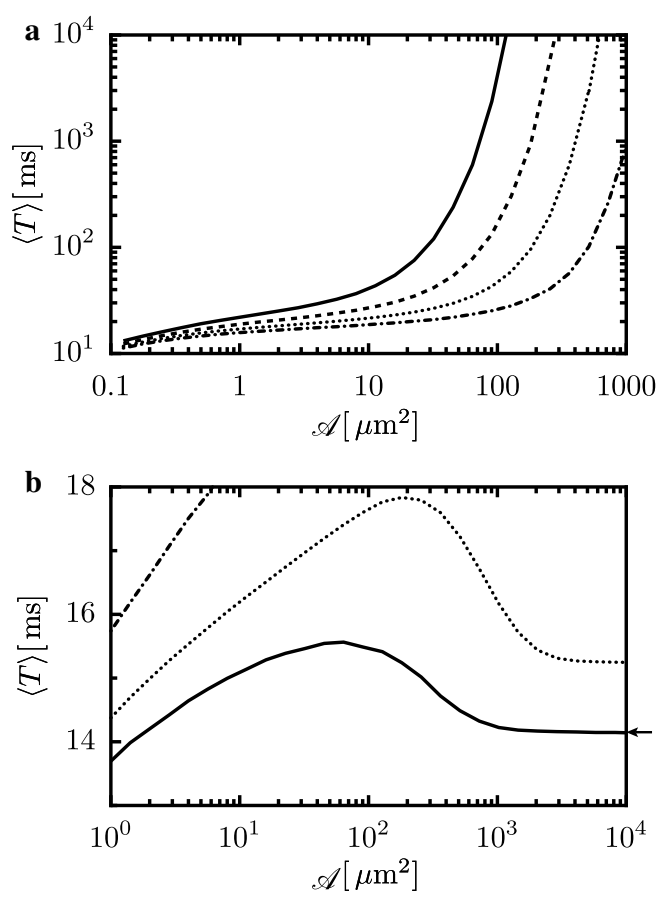

Fig. 4. The dependence of the mean interspike interval $\langle T\rangle$ vs. patch size $\mathscr{A}$ for sub-threshold constant driving currents $\left(I_{\text {ext }}<6.26 \mu \mathrm{A} / \mathrm{cm}^{2}\right.$ ) is depicted in (a): $I_{\mathrm{ext}}=0$ (solid line), $I_{\mathrm{ext}}=2 \mu \mathrm{A} / \mathrm{cm}^{2}$ (dashed line), $I_{\mathrm{ext}}=4 \mu \mathrm{A} / \mathrm{cm}^{2}$ (dotted line), $I_{\mathrm{ext}}=6 \mu \mathrm{A} / \mathrm{cm}^{2}$ (dash-dotted line), and for an intermediate driving $\left(I_{\mathrm{ext}}=9 \mu \mathrm{A} / \mathrm{cm}^{2}-\right.$ dotted line) and a strong driving $\left(I_{\mathrm{ext}}=11 \mu \mathrm{A} / \mathrm{cm}^{2}\right.$ - solid line) in (b). As a point of reference we depicted again the curve for the sub-threshold driving $I_{\text {ext }}=6 \mu \mathrm{A} / \mathrm{cm}^{2}$ (dash-dotted line) in (b). The small arrow placed to the right of (b) depicts the deterministic spiking period $\mathscr{T}$ for $I_{\mathrm{ext}}=11 \mu \mathrm{A} / \mathrm{cm}^{2}$.

driving. As a result, a bell-shaped behavior of the dependence of the mean interspike interval on the patch sizes is observed. The peak height is more pronounced for weak, but still supra-threshold driving. In case of an intermediate current driving, i.e., $\left(I_{1}<I_{\text {ext }}<I_{2}\right)$, for which both the fixed point and the spiking solution coexist, the limiting value of the spiking period in the deterministic limit $\mathscr{A} \rightarrow \infty$ depends on the size of the deterministic basins of attraction and the initial conditions, respectively (not shown).

For a sinusoidal driving, i.e., $I_{\text {ext }}:=A \sin (\Omega t)$, a synchronization of the firing events with the externally applied supra-threshold driving for large patch sizes could be observed. In contrast, for small membrane patches the channel noise reigns the spiking dynamics [13]. Due to skipping events, a perfect frequency synchronization could not be attained.

\section{Coherence resonance}

A quantity that sensitively measures the regularity of a spike train is the so-called coefficient of variation $(\mathrm{CV})$, or the relative dispersion of the interspike interval distribution. This regularitymeasure reads explicitly, 


$$
\mathrm{CV}:=\frac{\sqrt{\left\langle T^{2}\right\rangle-\langle T\rangle^{2}}}{\langle T\rangle} .
$$

It involves the mean interspike interval, given by Eq. (9), and the mean squared interval $\left\langle T^{2}\right\rangle:=\lim _{N \rightarrow \infty} \sum\left(t_{i+1}-t_{i}\right)^{2} / N$. For a fully uncorrelated sequence of spikes, which corresponds to the Poissonian spike train, this coefficient of variation would assume the value $\mathrm{CV}=1$. For a more ordered spike train, the coefficient of variation assumes values less then one, $\mathrm{CV}<1$. For a purely deterministic response, the $\mathrm{CV}$ equals zero.

Our Figs. 5 and 6 depict the coefficient of variation versus increasing system size $\mathscr{A}$. Whereas the firing rate decreases monotonically with respect to the patch area, the CV reveals the phenomenon of intrinsic coherence resonance [10]: at an optimal dose of internal noise, i.e., an optimal size of the cell membrane patch near $\mathscr{A}=1 \mu \mathrm{m}^{2}$, the $\mathrm{CV}$ exhibits a minimum, where the spike train becomes predominantly more ordered. The spiking activity possesses an internal rhythm which withstands the external disturbances. This phenomenon is revealed for any sub-threshold driving, but the optimal patch size increases, see in Fig. 5. This shift of the minimum of $\mathrm{CV}$ is due to the competition between externally applied stimulus and the influence of the intrinsic noise. In contrast, for strong supra-threshold driving, see in Fig. 6, one finds a crossover towards a deterministic firing dynamics when $\mathscr{A} \rightarrow \infty$ with a corresponding
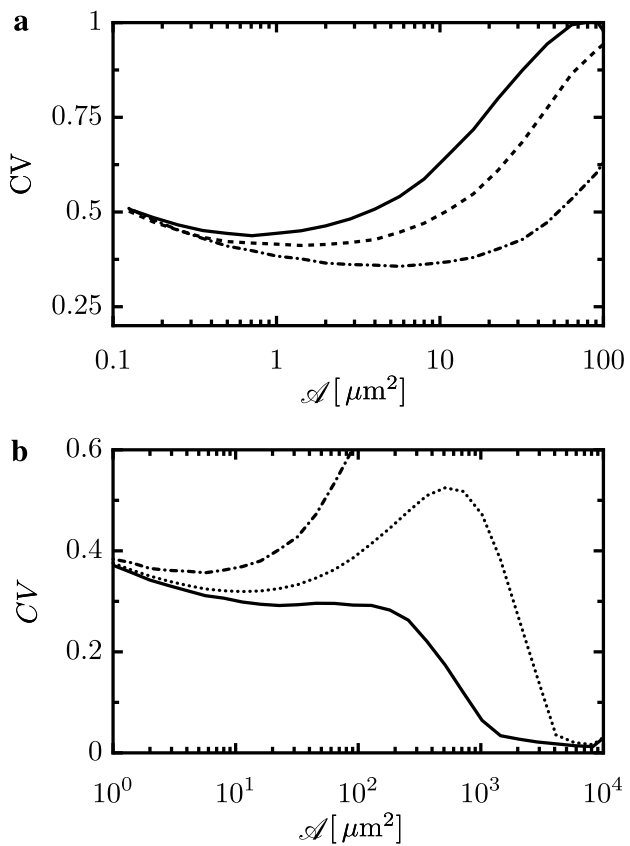

Fig. 5. The dependence of the coefficient of variation $C V$ in Eq. (10) vs. patch size $\mathscr{A}$ for sub-threshold constant driving currents $\left(I_{\mathrm{ext}}<6.26 \mu \mathrm{A} / \mathrm{cm}^{2}\right)$ is depicted in (a): $I_{\mathrm{ext}}=0$ (solid line), $I_{\mathrm{ext}}=2 \mu \mathrm{A} / \mathrm{cm}^{2}$ (dashed line), $I_{\mathrm{ext}}=4 \mu \mathrm{A} / \mathrm{cm}^{2}$ (dotted line), $I_{\mathrm{ext}}=6 \mu \mathrm{A} / \mathrm{cm}^{2}$ (dash-dotted line), and for an intermediate $\left(I_{\mathrm{ext}}=9 \mu \mathrm{A} / \mathrm{cm}^{2}-\right.$ dotted line) and a strong driving $\left(I_{\mathrm{ext}}=11 \mu \mathrm{A} / \mathrm{cm}^{2}\right.$ - solid line) in (b). As a point of reference we depicted again the curve for the sub-threshold driving $I_{\mathrm{ext}}=6 \mu \mathrm{A} / \mathrm{cm}^{2}$ (dash-dotted line) in (b). The coefficient of variation evidences the phenomenon of intrinsic coherence resonance for both, sub-threshold and supra-threshold driving. 


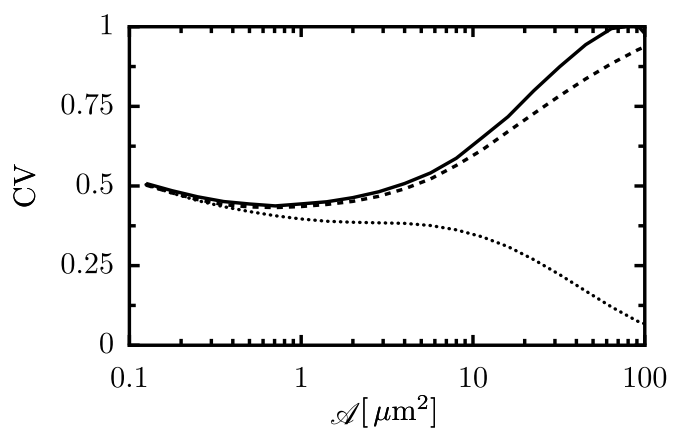

Fig. 6. The dependence of the coefficient of variation CV in Eq. (10) versus increasing patch size $\mathscr{A}$ for an oscillatory driving $I_{\text {ext }}=A \sin (\Omega t): A=0.0 \mu \mathrm{A} / \mathrm{cm}^{2}$ (solid line-absence of driving), $A=1.0 \mu \mathrm{A} / \mathrm{cm}^{2}, \Omega=0.3 \mathrm{~ms}^{-1}$ (dashed linebelow ac-threshold), and $A=3.0 \mu \mathrm{A} / \mathrm{cm}^{2}, \Omega=0.3 \mathrm{~ms}^{-1}$ (dotted line - above ac-threshold). Note, that the threshold amplitude for sinusoidal driving depends on the frequency. For $\Omega=0.3 \mathrm{~ms}^{-1}: A_{\text {threshold }}=1.55 \mu \mathrm{A} / \mathrm{cm}^{2}$. The phenomenon of intrinsic coherence resonance is observed.

vanishing of the coefficient of variation. However, for both, a constant and a sinusoidal, timedependent driving a maximum of the $\mathrm{CV}$ can be observed at intermediate channel noise strengths at near threshold driving strengths, note the dotted line in Fig. 6. In this regime, the noise does not reign the spiking behavior, but still hinders the deterministic spiking due to skipping events. The irregular spiking activity is more pronounced at weak supra-threshold signals. For strong driving this region of maximal disordered spiking could not be observed and a plateau-like structure arises. This indicates a direct cross-over from a totally noise-induced spiking behavior to a purely deterministic spiking regime with decreasing channel noise strength.

\section{Conclusions}

In summary, we have investigated the spiking activity in a noisy generalization of the HodgkinHuxley model, which incorporates a constant or sinusoidal time-dependent current driving and describes the role of spontaneous fluctuations of the membrane conductivity due to the individual ion channel dynamics - the so-called channel noise. The excitable membrane patch exhibits a noise-induced, rhythmic spiking activity at an optimal patch size. This effect can be regarded as intrinsic coherence resonance in presence of an optimal dose of intrinsic noise. In presence of an externally applied sub-threshold driving that is either constant or temporally sinusoidal, this phenomena is still detectable, although the most regular spiking behavior is shifted towards larger patch sizes. For weak supra-threshold driving, i.e., in presence of a stable and oscillatory spiking solution, a regime of patch sizes with worsening rhythmic spiking behavior shows up. This phenomena occurs due to the noise-induced skipping events at supra-threshold inputs. This behavior is accompanied by a maximum of the mean interspike period versus increasing patch size.

We share the confident belief that our investigations on the channel noise induced spiking activity in an archetypal model provide some new insights into the underlying physical principles and mechanism of neuronal signaling. 


\section{Acknowledgments}

The authors thank their collaborator Igor Goychuk for many fruitful discussions. This work has been supported by the Deutsche Forschungsgemeinschaft via the Sonderforschungsbereich SFB-486, Manipulation of matter on the nanoscale, Project A10.

\section{References}

[1] H. Lecar, R. Nossal, Theory of threshold fluctuations in nerves: I. Relationship between electrical noise and fluctuations in axon firing, Biophys. J. 11 (1971) 1048.

[2] H. Lecar, R. Nossal, Theory of threshold fluctuations in nerves: II. Analysis of various sources of membrane noise, Biophys. J. 11 (1971) 1068.

[3] J.A. White, J.T. Rubinstein, A.R. Kay, Channel noise in neurons, Trends Neurosci. 23 (2000) 131.

[4] J.R. Clay, L.J. DeFelice, Relationship between membrane excitability and single channel open-closed kinetics, Biophys. J. 42 (1983) 151.

[5] G. Schmid, I. Goychuk, P. Hänggi, Effect of channel block on the spiking activity of excitable membranes in a stochastic Hodgkin-Huxley model, Phys. Biol. 1 (2004) 61.

[6] E. Skaugen, L. Walløe, Firing behavior in a stochastic nerve membrane model based upon the Hodgkin-Huxley equations, Acta Physiol. Scand. 107 (1979) 343.

[7] L. Gammaitoni, P. Hänggi, P. Jung, F. Marchesoni, Stochastic resonance, Rev. Mod. Phys. 70 (1998) 223.

[8] P. Hänggi, Stochastic resonance in biology, ChemPhysChem 3 (2002) 285.

[9] P. Jung, J.W. Shuai, Optimal sizes of ion channel clusters, Europhys. Lett. 56 (2001) 29.

[10] G. Schmid, I. Goychuk, P. Hänggi, Stochastic resonance as a collective property of ion channel assemblies, Europhys. Lett. 56 (2001) 22.

[11] G. Schmid, I. Goychuk, P. Hänggi, S. Zeng, P. Jung, Stochastic resonance and optimal clustering for assemblies of ion channels, Fluct. Noise Lett. 4 (2004) L33.

[12] P.N. Steinmetz, A. Manwani, C. Koch, M. London, I. Segev, Subthreshold voltage noise due to channel fluctuations in active neuronal membranes, J. Comput. Neurosci. 9 (2000) 133.

[13] G. Schmid, I. Goychuk, P. Hänggi, Channel noise and synchronization in excitable membranes, Physica A 325 (2003) 165.

[14] J. Casado-Pascual, J. Gomez-Ordonez, M. Morillo, J. Lehmann, I. Goychuk, P. Hänggi, Theory of frequency and phase synchronization in a rocked bistable stochastic system, Phys. Rev. Rev. E 71 (2005) 011101.

[15] P. Talkner, L. Machura, M. Schindler, P. Hänggi, J. Luczka, Statistics of transition times, phase diffusion and synchronization in periodically driven bistable systems, New J. Phys. 7 (2005) 14.

[16] S.G. Lee, A. Neiman, S. Kim, Coherence resonance in a Hodgkin-Huxley neuron, Phys. Rev. E 57 (1998) 3292.

[17] H.C. Tuckwell, Spike trains in a stochastic Hodgkin-Huxley system, BioSystems 80 (2005) 25.

[18] A.S. Pikovsky, J. Kurths, Coherence resonance in a noise-driven excitable system, Phys. Rev. Lett. 78 (1997) 775 .

[19] R. Toral, C.R. Mirasso, J.D. Gunton, System size coherence resonance in coupled FitzHugh-Nagumo models, Europhys. Lett. 61 (2003) 162.

[20] M. Wang, Z. Hou, H. Xin, Optimal network size for Hodgkin-Huxley neurons, Phys. Lett. A 334 (2005) 93.

[21] A.L. Hodgkin, A.F. Huxley, A quantitative description of the membrane current and its application to conduction and excitation in nerve, J. Physiol. (London) 117 (1952) 500.

[22] B. Hille, Ion Channels of Excitable Membranes, 3rd ed., Sinauer Associates, Sunderland, MA, 2001.

[23] B. Sakmann, E. Neher (Eds.), Single-Channle recording, 2nd ed., Plenum Press, New York, 1995.

[24] B. Hassard, Bifurcation of periodic solutions of the Hodgkin-Huxley model for the squid giant axon, J. Theoret. Biol. 71 (1978) 401.

[25] J. Rinzel, R.N. Miller, Numerical calculation of stable and unstable periodic solutions to the Hodgkin-Huxley equations, Mathem. Biosci. 49 (1980) 27. 
[26] G. Schmid, I. Goychuk, P. Hänggi, Membrane clusters of ion channels: Size effects for stochastic resonance, Lect. Notes Phys. 625 (2003) 195.

[27] K. Aihara, G. Matsumoto, Y. Ikegaya, Periodic and non-periodic responses of a periodically forced HodgkinHuxley oscillator, J. Theor. Biol. 109 (1984) 249.

[28] R.F. Fox, Y.N. Lu, Emergent collective behavior in large numbers of globally coupled independently stochastic ion channel, Phys. Rev. E 49 (1994) 3421.

[29] P. Hänggi, H. Thomas, Stochastic processes: time-evolution, symmetries and linear response, Phys. Rep. 88 (1982) 207, see pp. 239-246.

[30] P. Hänggi, P. Jung, Colored Noise in Dynamical Systems, Adv. Chem. Phys. 89 (1995) 239.

[31] R.F. Fox, Contributions to the theory of multiplicative stochastic processes, J. Math. Phys. 13 (1972) 1196.

[32] W.H. Press, S.A. Teukolsky, W.T. Vetterling, B.P. Flannery, Numerical Recipes in C, 2nd ed., Cambridge Univ. Press, Cambridge, 1992. 